\title{
Casual sex and concurrent sexual partnerships among young people from an Yi community with a high prevalence of HIV in China
}

\author{
Yi Yang ${ }^{1,2}$, Rong-Sheng Luan ${ }^{1}$, Peng Liu ${ }^{1}$, Chun-Lin $\mathrm{Wu}^{1}$, Ying Zhou ${ }^{1}$ and Wen Chen ${ }^{1}$
}

Liangshan Prefecture in Sichuan province, China, has a high prevalence of HIV infection, which is reflective of a change in the mode of transmission from injection drug use (IDU) to heterosexual intercourse. However, few studies focus on HIV-related heterosexual risk behaviours among the majority Yi population. The objectives of this study were to explore the characteristics of an egocentric sexual network and estimate the prevalence of casual sexual behaviour. Yi villagers $(n=108)$, aged $15-35$ years, who reported having had sex within the previous year were interviewed as to their sexual behaviours and networks. In-depth interviews and focus group discussions provided supplementary information on sexual norms. Logistic regression analysis was used to calculate unadjusted odds ratios (ORs) and $95 \%$ confidence intervals (Cls). Most of the respondents reported having had casual sex at some time in their life, and $66.7 \%$ reported multiple sexual partnerships. Only $21.3 \%$ reported ever having used a condom. During the study year, a total of 137 partners were involved in 153 sexual partnerships. Among the reported sexual partnerships, $67.3 \%$ originated from a casual sexual relationship. For network members in components of size $\geqslant 3,56.9 \%$ were involved in concurrent sexual partnerships. Having never been married (OR: $2.11 ; 95 \% \mathrm{Cl}: 1.03-4.33$ ) and younger age (OR: $0.89 ; 95 \% \mathrm{Cl}: 0.83-0.95)$ were both associated with being in a component of size $\geqslant 3$. Size (OR: $2.99 ; 95 \% \mathrm{Cl}: 1.17-7.66$ ), pair (OR: $0.54 ; 95 \% \mathrm{Cl}: 0.39-0.74$ ), the number of weak components of the egocentric sexual network (OR: $30.04 ; 95 \% \mathrm{Cl}: 6.47-139.46$ ) and gender (OR: 0.19; $95 \% \mathrm{Cl}: 0.06-0.67$ ) were all associated with being in concurrent sexual partnerships. HIV-related interventions for the Yi ethnic minority in Sichuan province must therefore address concurrent sexual partnerships and promote condom use.

Asian Journal of Andrology (2012) 14, 758-765; doi:10.1038/aja.2012.25; published online 11 June 2012

Keywords: AIDS; China; concurrent sexual partnership; egocentric; ethnic minority; HIV; sexual behaviour; sexual network; Yi

\section{INTRODUCTION}

The HIV/AIDS epidemic in China was previously driven by transmission through injection drug use (IDU), but is now predominantly driven by heterosexual transmission. It remains an epidemic of low overall prevalence $(0.05 \%)$, although pockets of high infection among specific subpopulations exist in some localities. ${ }^{1}$ Liangshan Yi Autonomous Prefecture (Liangshan), Sichuan Province, exhibits both high prevalence and the change in transmission route. Analysis of surveillance data from Liangshan shows that members of the Yi ethnic minority accounted for the majority of infections and that, over time, the percentage of IDU accounting for HIV transmission decreased, whereas transmission by heterosexual sex increased., ${ }^{2,3}$

With a population of 2.31 million, the Yi ethnic group accounts for $48.85 \%$ of the total population in Liangshan, ${ }^{4}$ and Liangshan represents the largest Yi community in China. ${ }^{5}$ The Yi still maintain traditional social norms and values including arranged marriage between members of the same social status and condoned casual sex. ${ }^{6,7}$ People involved in casual sex do not describe it as either romantic or as involving a boyfriend or girlfriend, and it includes premarital sex and extramarital sex before luofujia (the time when a bride goes to live with her husband).
Explanations for variations in the timing and intensity of the HIV epidemic based on the rapid and pervasive spread among heterosexual populations in sub-Saharan Africa indicate that concurrent sexual partnerships exponentially increase the number of infected individuals and the growth rate of the epidemic during its initial phase. ${ }^{8}$ To identify concurrent sexual partnerships, sexual network analysis can be used. Sexual network analysis focuses on the sexual partnerships among individuals in a group using egocentric ${ }^{9-11}$ and sociometric $^{12-15}$ perspectives. Data for egocentric sexual networks can be collected anonymously.

Currently, few studies have focused on casual sex among the Yi in Liangshan. To explore the characteristics of an egocentric sexual network and estimate the prevalence of casual sex among the Yi, we conducted a survey of Yi villagers during December 2009.

\section{MATERIALS AND METHODS}

Data collection

Although China's HIV epidemic remains one of low overall prevalence, the rates in some counties in Liangshan (including County A) have risen rapidly. ${ }^{1}$ By the end of August 2009, surveillance data reported that there were 1164 cases of HIV-infected or AIDS patients

${ }^{1}$ West China Medical School of Public Health, Sichuan University, Chengdu 610041, China and ${ }^{2}$ Chengdu University of Traditional Chinese Medicine, Chengdu 611137, China Correspondence: Professor RS Luan (luan_rs@scu.edu.cn, luan_rs@163.com)

Received: 20 November 2011; Revised: 15 January 2012; Accepted: 15 February 2012; Published online: 11 June 2012 
living in County A, where the Yi comprise over 95\% of the rural population. ${ }^{16}$ The HIV prevalence rate in our study township of 14 villages ranks in the middle in County A.

During August 2009, we attempted to conduct a pilot survey using cluster sampling in two villages, but failed. Unmarried villagers either refused to take part in the survey or reported that they did not engage in sexual behaviour. Married villagers reported that they had taken part in sexual behaviour only with their spouses. After consulting with key informants, we determined that surveys regarding sexual behaviour were very sensitive issues among the Yi. As it is very difficult to use probability sampling, a combination of convenience sampling and snowball sampling was used in the current study.

The research sample consisted of $100 \mathrm{Yi}$ villagers between 15 and 35 years old who indicated having had sex in the previous year. We used the following formula for sample size

$$
n=u_{\alpha}^{2} \hat{P}(1-\hat{P}) N /\left[d^{2}(N-1)+u_{\alpha}^{2} \hat{P}(1-\hat{P})\right]
$$

where $n$ is the sample size, $\alpha$ is the size of a test, $\hat{P}$ is the estimation of population prevalence, $d$ is the maximum permissible error between the estimated value and the true value and $N$ is the total population number. In our study, $\hat{P}$ represented the estimation of the proportion of the population with multiple sexual partners among young Yi villagers; based on the literature, a value of $30 \%$ was used. ${ }^{17-20}$ For the remaining values, $d=0.1, N=71179, \alpha=0.05$ and $\mu_{\alpha}=1.96$. Therefore, $n$ was 81 . As the number of Yi between the ages of 18 and 35 years was 71179 , and because our study recruited Yi between the ages of 15 and 35 years, 100 individuals were ultimately selected.

First, the local rural doctor explained the study to the villagers, and the ones who agreed to take part in the study became the 'seeds' of the study; the seeds were then encouraged to bring their friends to participate. Recruitment was stopped when the number of interviewees reached 120; of these, 108 met the recruiting standards. Finally, 89 male and 19 female Yi between the ages of 15 and 35 years (representing eight villages) who reported having had sex in the previous year were interviewed. Face-to-face structured interviews using an anonymous questionnaire were conducted in private rooms at the township hospital by trained interviewers. Three male staff members from the County A Centres for Disease Control and Prevention (CDC), who were Yi and fluent in both Yi and Mandarin Chinese, were trained as interviewers and conducted part of the interviews. Additionally, one male and one female rural doctors, along with one female nurse from the township hospital, who were Yi and could speak Mandarin Chinese, were trained as assistant interviewers. With the aid of the assistant interviewers, one male and two female researchers from Sichuan University conducted part of the interviews. All the interviews were completed in one Ganchangri (Ganchangri is the day that villagers gather in a township. Some villagers bring agricultural and homemade goods to the market to sell and in turn buy consumer goods. Other villagers gather to mingle. Ganchangri is much like a village festival and is normally fixed according to the calendar, e.g., Ganchangri in our study site is the fourth, fourteenth and twentyfourth of every month). Ethical approval was obtained from the Institutional Review Board of Sichuan University (judgment reference number 2010008). As most of the respondents were illiterate, informed consent was administered orally in Yi before the interviews began. Those who refused to participate were not recruited. A name generator was used to investigate the egocentric network of Yi villagers using a recall period of the previous year. Respondents were asked to describe each of their partners in terms of current demographic information. Detailed information on their sexual partnerships was collected, including the dates of first and last sexual contact, number of sexual contacts, sexual partnership before first sex encounter and current sexual partnership, money exchanged and condom use. Participants were compensated with small gifts valued at 10 Chinese Yuan (CNY).

Additionally, a qualitative survey was conducted to collect supplementary information related to sexual norms and values among the Yi and related to the casual sexual experiences of young people, including contraceptive use and the methods used to avoid contracting HIV and STDs. The purpose of the qualitative survey was to compare and contrast different sources of information. With the aid of the assistant interviewers, two male and two female researchers from Sichuan University conducted the qualitative survey, and field notes were taken. In-depth interviews were conducted with six male and six female Yi villagers. Key informants (one village head, one village doctor, one township doctor and one staff member from the County A CDC) were also interviewed. Finally, one focus group discussion (FGD) among the local Bimo (A Bimo is an Yi shaman) and one FGD among village doctors were conducted.

\section{Data management and analysis}

Quantitative analysis. SPSS 18.0 (SPSS China, Shanghai, China) was used to conduct statistical analyses. Bivariate relationships between risk categories (such as whether an individual was in a concurrent sexual partnership) and variables of interest were analysed using nonparametric cross-tabulation ( $\chi^{2}$ and Fisher's exact test) for categorical data and $t$-test and ANOVA for continuous data. Logistic regression analysis was used for multivariate analysis, and non-significant explanatory variables $(P>0.20)$ were removed. Unadjusted odds ratios (ORs) and $95 \%$ confidence intervals (CIs) were calculated.

Sexual network analysis. Respondents and the sexual partners of the respondents were included as members in the sexual network. Two apparently independent partners mentioned by more than one respondent were considered the same individual, if all of the following characteristics matched: (i) gender, (ii) ethnicity, (iii) age, (iv) marital status, (v) education, (vi) occupation, and (vii) migration history. The current sexual partnership was used as an exclusion condition (more specifically, if the above seven characteristics matched and the current sexual partnership was anything other than 'husband and wife'). Concurrent sexual partnerships were also analysed. Definitions of concurrent sexual partnership currently share no consensus, as some exclude casual sex; ${ }^{21,22}$ in this study, concurrent sexual partnership refers to having multiple sexual partnerships, including casual sexual partnerships, overlapping within 1 month.

Ucinet 6.221 (Analytic Technologies, Lexington, KY, USA) was used to analyse the egocentric sexual network of network members. Parameters included size, pair, nWeakComp, pWeakComp, 2StepReach, ReachEffic, Broker and Normalized Broker. ${ }^{23}$

Qualitative analysis. Two interviewers read through all the transcripts and coded recurring themes together. Coding themes were determined by drawing upon the domains from qualitative guidelines and emergent information from transcripts. Particular themes, such as sexual norms and values, were identified and synthesized by the consistency and variability across groups (male and female) and between in-depth interviews and FGDs. The final stage 
Table 1 Demographic characteristics of the respondents and proxyreported sexual partners

\begin{tabular}{|c|c|c|c|c|}
\hline & \multicolumn{2}{|c|}{ Respondents } & \multicolumn{2}{|c|}{ Sexual partners } \\
\hline & No. & $\%$ & No. & $\%$ \\
\hline \multicolumn{5}{|l|}{ Gender } \\
\hline Male & 89 & 82.4 & 19 & 13.9 \\
\hline Female & 19 & 17.6 & 118 & 86.1 \\
\hline \multicolumn{5}{|l|}{ Marital status } \\
\hline Never married & 22 & 20.4 & 53 & 38.7 \\
\hline Married but living separately & 14 & 13.0 & 18 & 13.1 \\
\hline Married and living together & 69 & 63.9 & 53 & 38.7 \\
\hline Divorced & 3 & 2.8 & 1 & 0.7 \\
\hline Unknown & 0 & 0.0 & 12 & 8.8 \\
\hline \multicolumn{5}{|l|}{ Education } \\
\hline Illiterate & 74 & 68.5 & 91 & 66.4 \\
\hline Primary school & 26 & 24.1 & 18 & 13.1 \\
\hline Junior middle school & 4 & 3.7 & 12 & 8.8 \\
\hline Senior middle school/technical school & 4 & 3.7 & 7 & 5.1 \\
\hline Unknown & 0 & 0.0 & 9 & 6.6 \\
\hline \multicolumn{5}{|l|}{ Migration history } \\
\hline One year before & 40 & 37.0 & 10 & 7.3 \\
\hline Within the previous one year & 14 & 13.0 & 12 & 8.8 \\
\hline Never & 54 & 50.0 & 99 & 72.3 \\
\hline Unknown & 0 & 0.0 & 16 & 11.7 \\
\hline
\end{tabular}

of analysis involved translating the original quotes and preparing the results. ${ }^{24}$

\section{RESULTS}

\section{Characteristics of respondents}

Respondents were mostly male $(89,82.4 \%)$, illiterate $(74,68.5 \%)$, married and living with their spouse $(69,63.9 \%)$; 54 (50.0\%) had a history of migration (Table 1). The mean age of the participants was 27.5 years (interquartile range (IQR): 24.0-31.0), and the mean age at the time of marriage, for those who were married, was 21.7 years (IQR: 19.0-24.0). Of the respondents, $96.3 \%$ reported never using drugs.

\section{Sexual behaviour}

The mean age at sexual debut was 19.3 years for males (IQR: 17.520.5 ) and 22.0 years for females (IQR: 19.0-25.0, $P<0.01$ ). The mean age gap between marriage and sexual debut was 1.1 years, 2.4 years for males and -1.0 for females (Conventionally, a bride returns to the house of her parents after the wedding and may not have sex with her husband for years. In our study, the maximum delay was 5 years.) $(P<0.01)$. As a group, $89(82.4 \%)$ respondents reported having had casual sex at some time in their life, $72(66.7 \%)$ reported having had more than one partner, and 42 (38.9\%) reported having had more than four partners. The maximum number of partners was reported as 40. Males reported having had more sexual partners than females $(P<0.001$, Table 2$)$. Of the respondents, $52(58.4 \%)$ males and 2 $(10.5 \%)$ females reported having had casual sex at Ganchangri
$(P<0.01)$, and $48(53.9 \%)$ males reported having had sex when attending weddings $(P<0.01)$. Of the male respondents, $7(7.9 \%)$ reported having had commercial sex, but no females reported doing so $(P<0.001$, Table 2).

In the previous year, $22.2 \%$ of respondents reported having had multiple sexual partners, and $2.2 \%$ reported having had more than four partners, with the maximum number being 6 . Males reported having had more sexual partners than females in the past year $(P<0.001$, Table 2$)$. Of the female respondents, $10.5 \%$ reported having had five sexual partners accumulatively, and all of them reported having had only one sexual partner in the previous year.

\section{Condom usage}

In their lifetime, $51(47.2 \%)$ respondents reported having heard of condoms; among these, 28 (54.9\%) reported having never used them, $13(25.5 \%)$ reported using them occasionally and 10 (19.6\%) reported using them often. No one reported using them consistently. As a group, only $13(12.0 \%)$ reported using them occasionally, and 10 $(9.3 \%)$ used them often.

\section{Sexual network}

Characteristics of the sex partners of respondents. The respondents mentioned 153 sexual partnerships; nine sexual partners appeared repeatedly, one of whom was mentioned by six respondents. Thus, 137 sexual partners were involved in 153 sexual partnerships. Among the proxy-reported sexual partners, most were farmers $(117,85.4 \%)$, Yi $(134,97.8 \%)$, illiterate $(91,66.4 \%)$, and had never migrated (99, $72.3 \%)$, while $53(38.7 \%)$ were married and living together, 18 (13.1\%) were married and living separately, and 53 (38.7\%) had never married (Table 1). The mean age of the sexual partners was 26.8 years old (IQR: 23.0-26.0) (24 respondents were excluded due to lack of information.).

Types of sexual partnerships and condom use. Among all the 153 sexual partnerships, $67.3 \%$ were reported as beginning from casual sex, but only $45.8 \%$ were reported as casual sexual partnerships at the time of survey $(P<0.001)$. Moreover, among all the sexual partnerships, $96.1 \%$ were reported as non-commercial sex and $81.7 \%$ reported having never used a condom.

Sexual network. The whole network consisted of 153 sexual partnerships involving 108 respondents and 137 proxy-reported sexual partners (Figure 1). The average size of the egocentric network was 1.3, the average pair (pair refers to number of ordered pairs, which is the number of possible directed ties in each ego network) was 0.9 , and the average number of weak components (weak component is the largest number of actors who are connected, disregarding the direction of the ties) (nWeakComp) was 1.3 (Table 3). The network degree of centralisation was $2.0 \%$. The whole network consisted of 92 components, a component size heterogeneity of 1.0, and a fragmentation of 1.0.

Table 2 Comparisons of the number of sexual partners between males and females

\begin{tabular}{|c|c|c|c|c|c|c|c|c|}
\hline & \multicolumn{3}{|c|}{ No. of sexual partners for males $(n=89)$} & \multicolumn{3}{|c|}{ No. of sexual partners for females $(n=19)$} & $t^{a}$ & $\mathrm{P}$ \\
\hline Accumulatively & 89 & 7.6 & 8.6 & 19 & 1.4 & 1.3 & 6.4 & $<0.001$ \\
\hline Commercially within the past 1 year & 89 & 0.1 & 0.3 & 19 & 0.0 & 0.0 & 2.6 & $<0.001$ \\
\hline
\end{tabular}

${ }^{a}$ Welch's approximate $t$-test. 


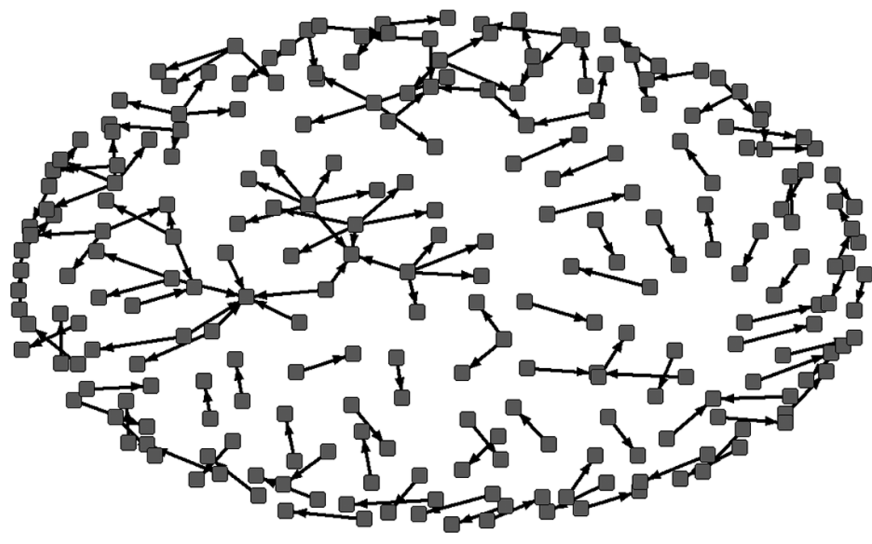

Figure 1 Whole sexual network for the previous year. Square nodes represent respondents and their partners. Lines represent sexual contacts.

Of the identified components, $82.6 \%$ were of size 2 but included only $62.0 \%$ of network members, while $17.4 \%$ were of size $\geqslant 3$ and included $38.0 \%$ of network members. The main component of size 28 includes $11.4 \%$ of network members. According to the size of the components, the whole network was divided into three types: small component, middle component and main component. Education level, marital status, occupation, and mean age in the component types were different (Table 4). Logistic regression analysis showed that never married (OR: 2.11 ; 95\% CI: $1.03-4.33$ ) and younger age (OR: 0.89; 95\% CI: 0.83-0.95) were associated with being in a component of size $\geqslant 3$ ( Table 5 ).

The main component consisted of $8(7.4 \%)$ respondents and 20 $(14.6 \%)$ sexual partners (Figure 2). All members of the main component were Yi, 19 (67.9\%) were male, 26 (92.9\%) were farmers, 20 (71.4\%) were illiterate, $23(82.1 \%)$ had a history of migration, and 17 $(60.7 \%)$ had never been married. The mean age of the members was 24.25 years old.

Concurrent sexual partnerships. Of all network members, $21.6 \%$ were involved in concurrent sexual partnerships at some time in the pre-

Table 3 Characteristics of the egocentric sexual network

\begin{tabular}{lrc}
\hline & $\bar{\chi}$ & $\mathrm{s}$ \\
\hline Size & 1.3 & 0.8 \\
Pair & 0.9 & 3.7 \\
nWeakComp & 1.3 & 0.8 \\
pWeakComp & 100.0 & 0.0 \\
2StepReach & 0.9 & 0.9 \\
ReachEffic & 100.0 & 0.0 \\
Broker & 0.5 & 1.8 \\
Normalized broker & 0.5 & 0.0 \\
\hline
\end{tabular}

Size of ego network is the number of nodes that one-step out neighboursof ego, plus ego itself. Pair refers to number of ordered pairs, which is the number of possible directed ties in each ego network. nWeakComp refers to number of weak components, and weak componentis the largest number of actors who are connected, disregardingthe direction of the ties. pWeakComp refers to number of weak componentsdivided by size. 2StepReach refers to number of nodes within two links to ego, that is two-step reach goes beyond ego's one-step neighborhood to report the percentage of all actors in the whole network that are within two directed steps of ego. ReachEffic refers to two-step reach divided by size. Broker refers to number of pairs notdirectly connected. Normalized Broker refers to brokerage divided bynumber of pairs. vious year, which was $56.9 \%$ of network members in components of size $\geqslant 3$. Of the respondents who accumulatively had multiple sexual partners, $62.5 \%$ were in concurrent sexual partnerships at some time in the previous year. Gender, marital status, ethnicity, migration history of members, size, Broker, pairs, nWeakComp and 2StepReach in concurrent sexual partnerships were different from those not in concurrent sexual partnerships (Table 6). Logistic regression analysis showed that size (OR: 2.99; 95\% CI: 1.17-7.66), pair (OR: 0.54, 95\% CI: 0.39-0.74), nWeakComp of the egocentric sexual network (OR: 30.04; 95\% CI: 6.47-139.46) and gender (OR: 0.19; 95\% CI: 0.06-0.67) were associated with being in concurrent sexual partnerships (Table 7).

\section{Results of qualitative data analysis}

Casual sex: frequently happens in daily life. Key informants estimated that the prevalence of casual sex among the Yi was over $60 \%$ and explained the remaining $40 \%$ as ' $50 \%$ of them were strictly forbidden by their families, $50 \%$ of them want to but can't , for example, men are poor or ugly looking and women are ugly looking'. One key informant described the attitude in the Yi society toward casual sex as 'can do but can't say', especially for women. Casual sex normally occurs between young people who know each other. Ganchangri and weddings provide opportunities for young people, especially those who are unmarried, to meet and have casual sex. Both in-depth interviews and FGDs showed that there is no word in the Yi language that means 'rape'. 'As long as both of them agree, they can have [sex]. If just one wants, it can't happen.' Both males and females agreed that 'usually boys go after girls'. One 18-year-old unmarried male informant said, 'When Ganchang, I usually go for women, and go for at every Ganchangri ... this year, I have done [had sex with] 40 or 50'. One male informant described the process as follows:

'Usually [we] know each other before, maybe not know well, but at least know. [At the Ganchangri], I shout at her and ask her to come to chat. I invite her to drink or eat candy and then make an agreement that we don't go home that night. During the night, it is ok to stay in the field or in an inn ... In [the twentieth century] '90s, men and women all played here, one man and one woman just leave together, it was more interesting than Ganchang.'

After that, they do not keep in touch with each other to avoid unnecessary trouble and possible conflict. One female informant said,

'If they are not arranged to get married and people know that they have had sex, the man has to pay a lot of money [to the family of the woman], especially when the man has had sex with a married/engaged woman.'

Married people usually only have sex with their spouses. However, some married men who are 'rich' and 'good-looking' and some married women who 'don't live with their husbands' are also involved in casual sexual partnerships. One married male informant said,

'If I go after a girl, she may think that he is married and still go out, [thinking that] there must be moral problem with him. Of course, the situation is based on a man's capacity. If a man in a family is not at home, [I] go and do [have sex with] a woman, there is no problem. The key point is a man's capacity.'

Men who have migrated are more 'attractive' than others and have more partners.

Condom use. In their FGD, Bimos said, 'Conventionally, there is no specific method for contraception.' Information from the FGD among village doctors showed that they do not teach villagers to use condoms at all. One village doctor said, 'as long as they can put on, it is ok', while 
Table 4 Characteristics of network members in different types of components

\begin{tabular}{|c|c|c|c|c|c|c|}
\hline & \multicolumn{3}{|c|}{ No. (\%) } & $\chi^{2}$ & $\mathrm{~F}$ & $P$ \\
\hline Male & 76 (70.4) & $23(21.3)$ & $9(8.3)$ & 5.77 & & 0.06 \\
\hline Female & $76(55.5)$ & $42(30.7)$ & 19 (13.9) & & & \\
\hline \multicolumn{7}{|l|}{ Education } \\
\hline Junior middle school & $7(43.8)$ & $8(50.0)$ & $1(6.2)$ & & & \\
\hline $\begin{array}{l}\text { Senior middle school } \\
\text { or technical school }\end{array}$ & $7(63.6)$ & $4(36.4)$ & $0(0.0)$ & & & \\
\hline Unknown & $0(0.0)$ & $5(55.6)$ & $4(44.4)$ & & & \\
\hline \multicolumn{7}{|l|}{ Migration history } \\
\hline One year before & 35 (70.0) & $10(20.0)$ & $5(10.0)$ & 12.49 & & 0.05 \\
\hline Married and living separately & $19(59.4)$ & $11(34.4)$ & $2(6.2)$ & & & \\
\hline Married and living together & $101(82.8)$ & $16(13.1)$ & $5(4.1)$ & & & \\
\hline Divorced & $3(75.0)$ & $0(0.0)$ & $1(25.0)$ & & & \\
\hline Unknown & $2(16.7)$ & $7(58.3)$ & $3(25.0)$ & & & \\
\hline \multicolumn{7}{|l|}{ Occupation } \\
\hline Farmer & $146(64.9)$ & $53(23.6)$ & $26(11.5)$ & & & $0.01^{\mathrm{a}}$ \\
\hline Other & $6(33.3)$ & $10(55.6)$ & $2(11.1)$ & & & \\
\hline Unknown & $0(0.0)$ & $2(100.0)$ & $0(0.0)$ & & & \\
\hline \multicolumn{7}{|l|}{ Ethnicity } \\
\hline Yi & $151(62.4)$ & $63(26.0)$ & $28(11.6)$ & & & $0.24^{\mathrm{a}}$ \\
\hline Han & $0(0.0)$ & $2(100.0)$ & $0(0.0)$ & & & \\
\hline Other & $1(100.0)$ & $0(0.00)$ & $0(0.00)$ & & & \\
\hline
\end{tabular}

${ }^{\text {a }}$ Fisher's exact test.

another village doctor said, 'They all know how to use [condoms]; nowadays, young people know things early'.

Young, unmarried Yi men give spare condoms to their friends. Some Yi men visited female sex workers when they migrated and learned how to use condoms during these encounters. For women, condoms were considered more as a contraceptive than as a tool to prevent STDs. One female informant said that the function of a condom was 'for contraceptive use, I don't know others'. Even married women were shy to accept condoms; one married woman said, 'In hospitals, people (doctors) asked whether I need condoms or not, I said no.'

Even though women expressed interest in using condoms during vaginal intercourse, men rather than women decide whether a condom will be used. One male informant said, 'girls dare not ask me to use a condom because we believe in each other .... I don't use a condom.' The distribution of free condoms from the family plan-

Table 5 ORs for being in components of size $\geqslant 3$

\begin{tabular}{lcc}
\hline & $O R$ & $95 \% \mathrm{Cl}$ \\
\hline Education (literate vs. illiterate) & 1.92 & $1.00-3.70$ \\
Marital status (never married vs. had been married) & 2.11 & $1.03-4.33$ \\
Age & 0.89 & $0.83-0.95$ \\
\hline
\end{tabular}

Abbreviations: $\mathrm{Cl}$, confidence interval; OR, odds ratio. ning bureaus only covers married people, thus missing many of the unmarried young people. Moreover, condoms are not widely sold in the townships, and those condoms that are available are sold in a box of ten at a price of $10 \mathrm{CNY}$ or higher, which is not cheap for the Yi.

\section{DISCUSSION}

A young person's lifestyle: casual sex

This study was based on non-IDU respondents. Although only 19 females were interviewed directly, we obtained information on young Yi women indirectly through the analysis of the whole sexual network. By combining qualitative with quantitative surveys, this study showed the prevalence of casual sex among Yi young people in a single township with a mid-level prevalence of HIV. We found that the Yi start to have casual sex when they are young. Some of these casual sexual partnerships later become married-to-be, and some Yi have only one sexual partner over the course of their entire life. When the Yi marry, they have fewer sexual partners than before, although some are involved in extramarital partnerships. This phenomenon, where some casual sexual partnerships become married-to-be, is in fact a revolution against arranged marriages that has occurred since the democratic reform. ${ }^{7}$ Casual sex, which occurs in the context of their social activities such as Ganchang, is therefore part of the Yi lifestyle. 


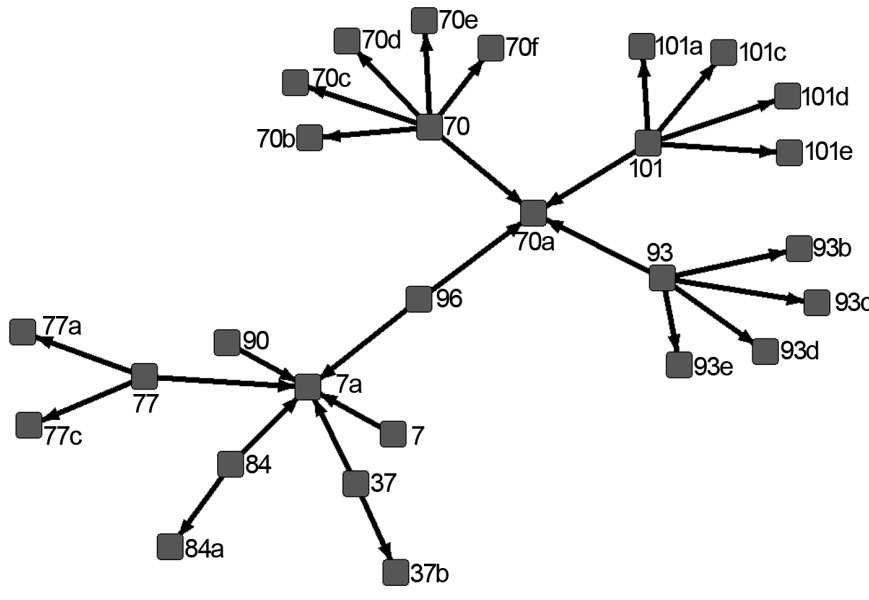

Figure 2 The main component including 28 members (respondents 7, 37, 70, $77,84,90,93,96$ and 101) along with their proxy-reported sexual partners. Arabic numbers and small English letters were used for coding. For example, 77a refers to the individual who was mentioned by respondent 77 as the first sexual partner in 77's sexual egocentric network. If one member was mentioned by more than one respondent, only one coding was used in accordance with his/ her coding the first respondent who mentioned him/her. For example, the individual who was mentioned by 7, 37, 70 and 90 as their first sexual partner respectively, and by 77 and 84 as their second sexual partner, respectively, was coded as 7a. Arrows refer to mentioned partners.

\section{A denied phenomenon: casual sex}

Due to the influence from the Han culture, which scolds those engaging in casual sex, the Yi feel sensitive when Han Chinese discuss casual sexual behaviour among them. The real meaning of the saying 'can do but can't say' is that they can have casual sex, but they cannot tell the Han. The reason this topic is so sensitive is related to the changing nature of HIV transmission in Liangshan.

The first case of HIV in Liangshan was detected in 1995. Surveillance data showed that between 1995 and 2000, 96.0\% of HIV transmission was due to IDU. ${ }^{2}$ Consequently, this is where HIV intervention policies focused. ${ }^{25}$ However, these interventions have not been successful, and the prevalence rate in some counties (including County A) in Liangshan has risen rapidly. ${ }^{1}$ In Liangshan, comparisons of surveillance data from 1995 to 2000 and from 1995 to 2007 showed that the percentage of IDU transmission has decreased from $96.0 \%$ to $46.3 \%$, while sexual transmission has increased from $0.0 \%$ to $2.3 \%$ (other modes of transmission remain unknown, but increased from $4.0 \%$ to $50.9 \%)^{2,3}$ We hypothesize that the unknown $50.9 \%$ represent either denied sexual behaviours, including casual sex, or a mixture of IDU and sexual behaviours. Unfortunately, no studies have been performed to verify this hypothesis, even though in 2003, Mo SD, who is on staff at the Liangshan CDC and who is also Yi, indicated his concerns over the potential role of casual sex in the HIV epidemic among Yi people. ${ }^{6}$

\section{Inconsistent condom use}

In sub-Saharan Africa, among those who predominantly had noncommercial sex, it has been shown that as the number of sexual partners increased, so did the incidence of HIV. ${ }^{13}$ This risk increases with multiple sexual partnerships and inconsistent condom use, and people with more than four sexual partners are at higher risk than those with fewer partners. ${ }^{26-30}$ Experiences in Uganda have shown that the decline in HIV prevalence since the early 1990s is linked to a reduction in casual sex, which is similar to the impact of a vaccine with $80 \%$ effectiveness. ${ }^{28,29}$ In simple terms, casual sex is linked to HIV prevalence. Studies in China showed that casual sex within the last 3 months was an independent risk factor for the acquisition of an STD. ${ }^{31}$ The most effective method available for protection against STDs is the condom. ${ }^{32}$ Condoms decrease the per-contact probability of male-to-female transmission of HIV by approximately $95 \% .{ }^{33}$ SubSaharan Africa still has the highest prevalence of HIV/AIDS in the world, because condom use remains low in many areas. ${ }^{34}$ In South Africa, where $15 \%$ of young women and almost $5 \%$ of young men aged 15-24 years were infected with HIV, the use of condoms at a proportion approximating $50 \%$ is lower than what would be necessary to curb the HIV/AIDS epidemic. ${ }^{35}$

In our study, 38.9\% of Yi young people reported having had more than four partners. The problem is not casual sex itself, but rather the inconsistent use of condoms. The proportion of condom use in our study was $21.3 \%$, which is lower than the proportion found in rural areas with high HIV/AIDS prevalence in China; ${ }^{17}$ the proportion is higher than before implementation of HIV intervention programs, but lower than after received HIV intervention in other Yi areas. ${ }^{17-20}$ Moreover, no one in our study reported consistent condom use. One study showed that even among university/college students in Shanghai, only $38.4 \%$ of respondents had received reproductive health education previously, and $55.7 \%$ knew when to use a condom. ${ }^{36}$ For the Yi villagers in our study, there are ideological, geographical and financial barriers to condom access.

\section{Increased risk due to concurrent sexual partnership}

Sexual network analysis indicated that $38.0 \%$ of network members were involved in components of size $\geqslant 3$, that is, they or their partners had multiple sexual partners in the previous year. Members who were younger and who had never married were apt to be in components of size $\geqslant 3$, which put them at a high risk for HIV even if they themselves had only one partner. ${ }^{34}$ More than half of the network members with multiple sexual partners in the previous year were in concurrent rather than serial sexual partnerships. In other words, Yi people tend to have concurrent sexual partnerships when they have multiple sexual partners. The proportion of concurrent sexual partnerships was even higher than among some parts of sub-Saharan Africa, ${ }^{37,38}$ which puts young Yi villagers from a community with high infection rates at great potential risk for acquiring HIV., 89

\section{Limitations of this study}

Our study had several limitations. First, surveys on sexual behaviour are a very sensitive issue among the Yi; thus, instead of probability sampling, a combination of convenience sampling and snowball sampling was used. More males than females were recruited, and thus, the results may not be representative of all the Yi living in Liangshan. Based on our pilot survey, we found that probability sampling could not be performed. Thus, this study was carefully designed to combine qualitative and quantitative surveys to best show the prevalence of casual sex among Yi young people in a single township with mid-level HIV prevalence. In the questionnaire survey, only $17.6 \%$ of females were recruited, but in the sexual network analysis, detailed information on respondents' sexual partnerships was collected. Overall, a total of 108 male and 137 female sexual network members were included in the analysis, thus compensating for the lack of female respondents.

Second, all variables were self-reported, and thus, information about sexual behaviour could be under-reported, especially among females. We used the following strategies to diminish under-reporting. First, all surveys were conducted either by trained interviewers who 
Table 6 Characteristics of members in and out of concurrent sexual partnerships

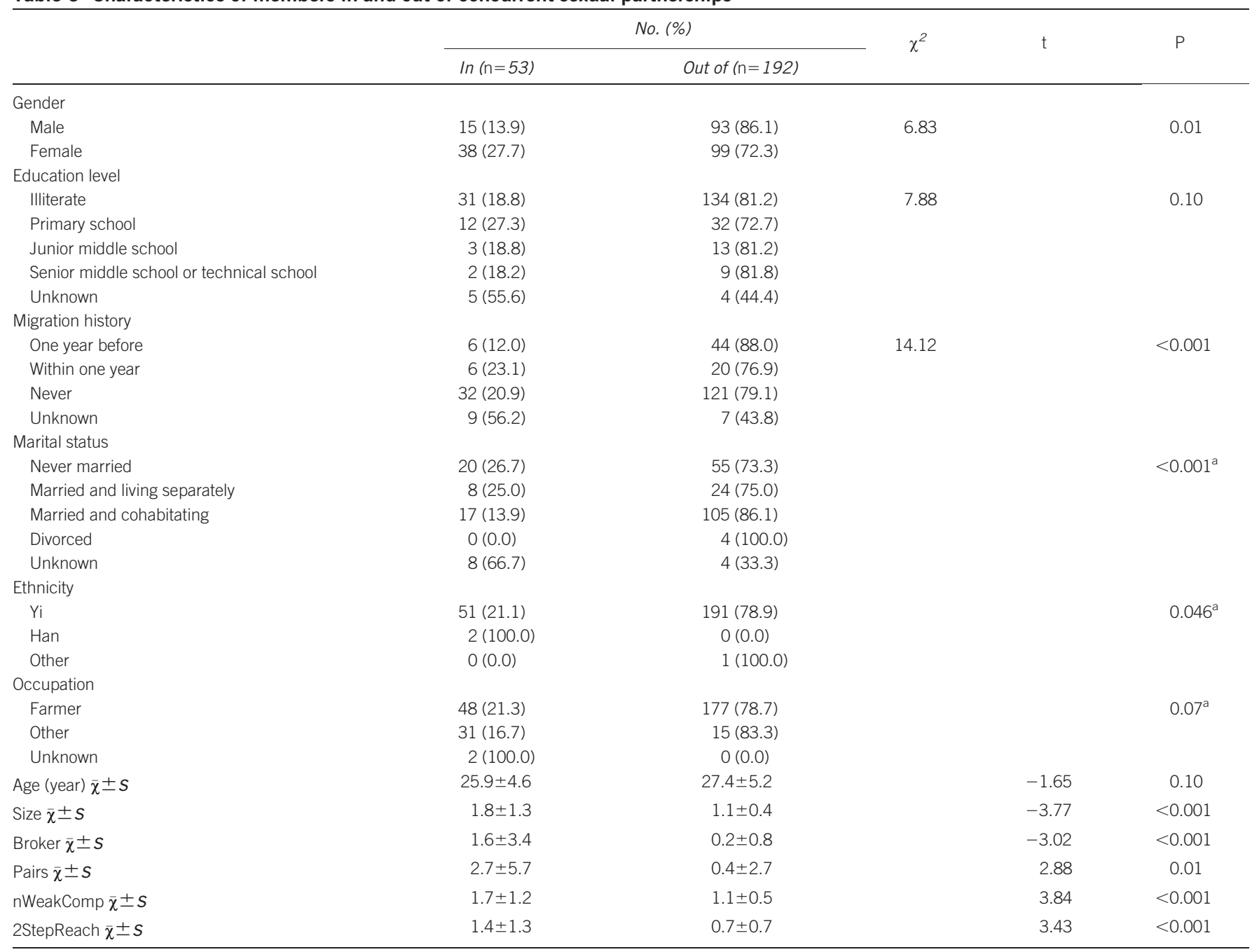

${ }^{a}$ Fisher's exact test.

were from the Yi ethnic group and fluent in both Yi and Mandarin Chinese or by trained interviewers with the help of assistant interviewers to avoid possible under-reporting from Yi respondents to Han interviewers. Second, all the interviewers were of similar age as the respondents; this was done to avoid possible under-reporting from younger respondents to older interviewers. Third, local idioms and metaphors were used to refer to sexual behaviour, thus avoiding embarrassment between respondents and interviewers. Fourth, face-to-face structured interviews using an anonymous questionnaire were conducted separately and in private rooms, with no one except an interviewer and a respondent present, to

Table 7 ORs for concurrent sexual partnerships

\begin{tabular}{lrrr}
\hline & & \multicolumn{2}{c}{$95 \% \mathrm{Cl}$} \\
\cline { 3 - 4 } & OR & Lower & Higher \\
\hline Size & 2.99 & 1.17 & 7.66 \\
Pairs & 0.54 & 0.39 & 0.74 \\
nWeakComp & 30.04 & 6.47 & 139.46 \\
Gender (male vs. female) & 0.19 & 0.06 & 0.67 \\
\hline
\end{tabular}

Abbreviations: $\mathrm{Cl}$, confidence interval; OR, odds ratio. guarantee confidentiality. Fifth, informed consent was administered orally in Yi before the interviews began. Those who refused to participate were not recruited. Respondents were encouraged to bring their friends to participate in the research because by doing so it was thought that these respondents would be more open to talking about their sexual experiences.

\section{CONCLUSION}

Casual sex is a part of young person's lifestyle among the Yi, but it is denied. Moreover, condom use among Yi young people is inconsistent. Concurrency rather than sequential monogamy puts young Yi villagers from a community with high prevalence at great potential risk for HIV infection.

Currently, there are few studies focusing on casual sex among the Yi in Liangshan, because it is either taken for granted or avoided under the name of 'harmony consideration of National Unity'. After studying the potential causes of the HIV epidemic in Liangshan, we advocate changing the current HIV-related behaviour intervention policies, which are mainly copied from the Han areas, because they do not give priority status to the unique culture of the Yi. Other than IDU, more research should be performed to determine the potential causes 
of the HIV epidemic in Liangshan and to obtain enough evidence to call attention of policy makers to the problem.

\section{AUTHOR CONTRIBUTIONS}

YY helped supervise the fieldwork, designed the study's analysis plan, and drafted the manuscript. RSL designed the study, directed its implementation, including quality assurance and control, and reviewed the manuscript. PL helped conduct the literature review and prepared the Materials and Methods section of the manuscript. CLW and YZ conducted the fieldwork and reviewed the manuscript. WC participated in the analysis and interpretation of data. All authors read and approved the final manuscript.

\section{COMPETING FINANCIAL INTERESTS}

We have no competing financial interests related to the material in the manuscript.

\section{ACKNOWLEDGMENTS}

This study was supported by the Sichuan Branch of the Global Fund AIDS Office (Round 4). We thank the individuals who made this research possible, particularly Qingguo Luo, Qiang Xu, Ladu AKu, Jian Xu and Qiujian Yang. We also thank Willa Dong for editorial assistance.

1 Press Offfice of Ministry of Health. [HIV/AIDS Epidemic in China]. Cap J Public Health 2010; 14: 1. Chinese.

2 Han YH, Gong YH. [Survelliance Data Analysis about AIDS from Year 1989 to 2000 in Liangshan Yi Autonomous Prefecture, Sichuan, China]. J Prev Med Inform 2002; 18 324. Chinese

3 Mo SD, Wang Z, Deng F, Zhang JH, Gong YH et al. [Analysis of epidemiologica characteristics and countermeasure of AIDS in Liangshan State]. Mod Prev Med. 2008; 35: 630-1, 5. Chinese.

4 [Annual bulletin of financial and social development in Liangshan Prefecture]. Bureau of Statistics of Liangshan Yi autonomous prefecture, 2010. http://www.tjcn.org/plus/ view.php?aid=10586. Chinese.

5 Survey of Liangshan. The People's Government of Liangshan Yi Autonomous Prefecture; 2010. http://www.Isz.gov.cn/tzzn-en.

6 Mo SD, Zhang JH, Gong YH. [HIV/AIDS related challenges and threats toward traditional sexual customs of Yi]. J Prev Med Inform 2003; 19(Suppl): 91. Chinese.

7 Highfill DM. Relationship of Knowledge to Perceived Risk of Susceptibility Awarenes of $\mathrm{Yi}$ (Nuoso) Women of Childbearing Age Located in Sichuan Province. Touro University International; 2004.

8 Morris M, Kretzschamar M. Concurrent sexual partnerships and the spread of HIV. AIDS 1997; 11: 641-8.

9 Potterat JJ, Muth SQ, Rothenberg RB, Zimmerman-Rogers H, Green DL et al. Sexual network structures as an indicator of epidemic phase. Sex Transm Infect 2002 78(Suppl 1): i152-8.

10 Choi K-H, Ning Z, Gregorich SE, Pan Q-C. The influence of social and sexual networks in the spread of HIV and syphilis among men who have sex with men in Shanghai, China. J Acquir Immune Defic Syndr 2007; 45: 77-84.

11 Remple VP, Patrick DM. Johnston C, Tyndall MW, Jolly AM. Clients of indoor commercial sex workers heterogeneity in patronage patterns and implications for HIV and STI propagation through sexual networks. Sex Transm Dis 2007; 34: 754 60.

12 Kretzschmar M, Morris M (eds). Measures of Concurrency in Networks and the Spread of Infectious Disease. New York: Elsevier Science Inc; 1995.

13 Helleringer SP, Kohler H-P. Sexual network structure and the spread of HIV in Africa: evidence from Likoma Island, Malawi. AIDS 2007; 21: 2323-32.
14 Latora V, Nyamba A, Simpore J, Sylvette B, Diane S et al. Network of sexual contacts and sexually transmitted HIV infection in Burkina Faso. J Med Virol 2006; 78: 724-9.

15 Friedman SR, Neaigus A, Jose B, Curtis R, Goldstein M et al. Sociometric risk networks and risk for HIV infection. Am J Public Health 1997; 87: 1289-96.

16 West China Medical School of Public Health SU, Meigu Center of Disease Prevention and Control L. [Report of an Epidemiological Survey of HIV/AIDS in Meigu, Liangshan]. Chengdu: West China Medical School of Public Health, Sichuan University; 2009. p28. Chinese.

17 Huang N, Chen YM, Li Y, Guo X, Wu JQ et al. [A cross-sectional survey of HIV/AIDS related knowledge and condom use in rural areas]. Chin J Behav Med Sci 2006; 15 165-6. Chinese.

18 Chen HG, Yu HT, Wang YN, Zhang XF, Yang Q et al. [The baseline investigation about information of preventing AIDS in population of $\mathrm{Yi}$ nationality for later health education]. J North Sichuan Med Coll 2005; 20: 75-8. Chinese.

$19 \mathrm{Hu}$ TB, Huang Y, Yin YZ, Chen XY, Lai HB et al. [A survey of HIV/AIDs related knowledge and behaviour in Yi areas in Mabian County]. J Prev Med Inform 2007; 23: 344-6. Chinese.

20 Wang S, Keats D. Developing an innovative cross-cultural strategy to promote HIV/ AIDS prevention in different ethnic cultural groups of China. AIDS Care 2005; 17 874-91.

21 Mah T, Halperin D. Concurrent sexual partnerships and the HIV epidemics in Africa evidence to move forward. AIDS Behav 2010; 14: 11-6.

22 Lurie M, Rosenthal S. Concurrent partnerships as a driver of the HIV epidemic in subSaharan Africa? The evidence is limited. AIDS Behav 2010; 14: 17-24.

23 Hanneman RA, Riddle M (eds). Introduction to Social Network Methods. Riverside CA: University of California; 2005. pp131-43.

24 Yang C, Latkin CA, Liu P, Nelson KE, Wang C et al. A qualitative study on commercia sex behaviors among male clients in Sichuan Province, China. AIDS Care 2010; 22 246-52.

25 Administrative Rules on the Prevention and Treatment of HIV/AIDS of Liangshan $Y$ Autonomous Prefecture, No. 24 Government Order of Liangshan Yi Autonomous Prefecture; 2008.

26 Agha S. Declines in casual sex in Lusaka, Zambia: 1996-1999. AIDS2002; 16: 291 3.

27 Munguti K, Grosskurth H, Newell J, Senkoro K, Mosha F et al. Patterns of sexual behavior in a rural population in north-western Tanzania. Soc Sci Med 1997; 44 1553-61.

28 Stoneburner R, Low-Beer D. Population-level HIV declines and behavioral risk avoidance in Uganda. Science 2004; 304: 714-8.

29 Asiimwe-Okiror G, Opio AA, Musinguzi J, Madraa E, Tembo G et al. Change in sexual behaviour and decline in HIV infection among young pregnant women in urban Uganda. AIDS 1997; 11: 1757-63.

30 Boileau C, Zunzunegui M, Rashed S. Gender differences in unsafe sexual behavior among young people in urban Mali. AIDS Care 2009; 21: 1014-24.

31 Lu F, Jia Y, Bin S, Li C, Limei S et al. Predictors for casual sex and/or infection among sexually transmitted disease clinic attendees in China. Int J STD AIDS 2009; 20: 241-8.

32 Da Ros CT, Schmitt Cda S. Global epidemiology of sexually transmitted diseases. Asian J Androl 2008; 10: 110-4.

33 Pinkerton SD, Abramson PR. Effectiveness of condoms in preventing HIV transmission. Soc Sci Med 1997; 44: 1303-12.

34 HIV/AIDS JUNPo, Organization WHO. 09 AIDS Epidemic Update: Joint United Nations Programme on HIV/AIDS. Geneva: World Health Organization; 2009. pp21-36.

35 Hendriksen E, Pettifor A, Lee S, Coates T, Rees H. Predictors of condom use among young adults in South Africa: the Reproductive Health and HIV Research Unit National Youth Survey. Am J Public Health 2007; 97: 1241-8.

36 Chen B, Lu Y-N, Wang H-X, Ma Q-L, Zhao X-M et al. Sexual and reproductive health service needs of university/college students: updates from a survey in Shanghai, China. Asian J Androl 2008; 10: 607-15.

37 Mah TL. Prevalence and correlates of concurrent sexual partnerships among young people in South Africa. Sex Transm Dis 2010; 37: 105-8.

38 Lagarde E, Auvert B, Carael M, Laourou M, Ferry B et al. Concurrent sexua partnerships and HIV prevalence in five urban communities of sub-Saharan Africa. AIDS 2001; 15: 877-84.

39 Morris M. Barking up the wrong evidence tree. Comment on Lurie \& Rosenthal, "Concurrent partnerships as a driver of the HIV epidemic in sub-Saharan Africa? The evidence is limited'. AIDS Behav 2010; 14: 31-3. 